\title{
Overview on Common Respiratory Virus
}

\author{
P. Sagadevan ${ }^{1}$, R. Ramya ${ }^{2}$, S. Rathishkumar ${ }^{1}$, S.Vijayakumar ${ }^{4}$, L Surendran ${ }^{1}$, P. Janarthanan ${ }^{1}$ and C. Sajith ${ }^{3}$ \\ Department of Biotechnology, Kongunadu Arts and Science College, Coimbatore \\ King Institute of Preventive Medicine and Research, Guindy, Chennai \\ Medgenome Pvt Ltd, Bangalore
}

Abbes Biotech, Kolathur Chennai

\begin{abstract}
Respiratory viruses have been recognised as causative agents for a wide spectrum of clinical manifestations and severe respiratory compromise in neonates during birth hospitalisation. Early-life respiratory virus infections have also been shown to be associated with adverse long-term consequences. Preventing virus infections by intensifying hygiene measures and cohorting infected infants should be a major goal for neonatal intensive care units, as well as more common use of virus diagnostics.
\end{abstract}

Key words: Acute tract respiratory infections, respiratory virus

\section{INTRODUCTION}

Acute respiratory tract infections are estimated to cause approximately 1.9 million childhood deaths annually (Williams et al., 2002). The contribution of acute respiratory infections to overall childhood mortality ranges from $<5 \%$ in the developed countries to $25 \%$ in some developing countries. Bacterial infections in general have a higher case fatality than acute viral infections, but viruses are far more common causes of acute respiratory infection. Overall they contribute to at least one-third of the deaths caused by acute respiratory infection in the developing world (Berman, 1991).

The respiratory tract can be divided into upper and lower parts, with the boundary at the lower end of the larynx. Viral infections confined to the upper part (upper respiratory tract infection, URTI) are rarely life-threatening, with the exception of croup. They can be uncomfortable but do not usually call the individual's future into question. These infections do not automatically spread to the lower respiratory tract, but where the lower respiratory tract is involved the process is extensive and rarely confined to one lobe or even one lung. This contrasts with pneumococcal pneumonia, which is typically confined to one lobe of one lung.

\section{RESPIRATORY VIRUSES}

Timely and accurate identification of respiratory viruses is rapidly becoming more relevant as antiviral treatment options increase. In addition, the resulting improved treatment of patients presenting with respiratory illness will help control infection, prevent nosocomial spread, and reduce patient stay as well as hospital costs (Sonia Letant et al., 2007). Traditionally, Respiratory Syncytial Virus (RSV), parainfluenza virus (PIV), influenza virus, and adenovirus have been viewed as being the leading causes of acute viral lower respiratory tract infections (LRTIs) (Henderson et al., 1979; Hall et al., 1990; Yun et al., 1995; Hong et al., 2001).

\section{RESPIRATORY SYNCYTIAL VIRUS}

Respiratory Syncytial Virus (RSV) is an envelope, negative-sense RNA virus belonging to the Paramyxoviridae family. It is the major cause of lower respiratory tract illness in young children (Hall, 2001). In recent years it has been recognized that RSV infection may be severe in certain adult populations, including the elderly, persons with cardiopulmonary disease, and the immuno compromised (Englund et al., 1988). Diagnosis of RSV infection by either culture or antigen detection from nasopharyngeal specimens is difficult with adults, presumably due to low viral titres in secretions (Falsey et al., 1996).

This virus is distributed worldwide and is found wherever it has been sought. It is frequently associated with bronchiolitis in babies - with a peak incidence at about 6 months - and is the most common virus detected,

\section{Corresponding author}

Dr. P. Sagadevan

Email address: sagadevan.biotech@gmail.com

Received: $30-03-2018$ 
especially in children under 1 year of age who are hospitalized with respiratory infections. Large epidemics occur annually at the same season, but the seasonality of RSV epidemics may vary in different geographical regions. The starting date and extent of the epidemic may vary but the annual epidemic occurs reliably. For diagnostic purposes there is only one serotype, but two subtypes (A and B) have been described and they may co-circulate, with one usually predominating in any given year. No obvious differences in disease severity or pathogenesis have been documented. RSV causes a substantial but variable LRTI disease burden in tropical countries (Robertson et al., 2004). In a population-based study of infants in Kenya it was found that RSV was common, approximately $36 \%$ of infections led to LTRI, 23\% were severe and $3 \%$ of infected children were hospitalized (Nokes et al., 2004).More recently, it is becoming clear that RSV causes significant morbidity in the elderly as well as in infants (Falsey et al., 2006).

\section{INFLUENZA A AND B VIRUSES}

Antigenically, these are the most variable of the respiratory viruses. Both exhibit antigenic 'drift', in which the surface antigens of the virus change gradually in the face of immunological pressure from the host species, with one or two variants predominating at a given time. In showing this progressive and 'directional' antigenic change, they are unique among respiratory viruses. In addition, influenza A, but not influenza B, shows occasional major antigenic changes in the surface antigenic structures (haemagglutinin and or neuraminidase) and called 'antigenic shift', which may lead to a pandemic. Such pandemic influenza viruses are derived from avian influenza viruses through genetic reassortment with animal or human strains. This results in the incorporation of new viral surface antigens to which the human population is immunologically native. The timing, extent and direction of either 'drift' or 'shift' have so far been completely unpredictable. However, when viruses with antigenic shift appear in the human population, a worldwide pandemic of influenza A becomes possible; memorable examples occurred in 1918 ('Spanish flu'), in 1957 ('Asian flu') and in 1968 ('Hong Kong flu'). With no animal reservoirs to provide such new antigens, shift does not occur in influenza B (Malik et al., 2008).

Acute lower respiratory tract infections (ALRI) are the major cause of morbidity and mortality in young children worldwide. Information on viral etiology in ALRI from India is limited. The study was done to develop a simple, sensitive, specific and cost effective multiplex PCR (mPCR) assay without post PCR hybridization or nested PCR steps for the detection of respiratory syncytial virus
(RSV), influenza viruses, parainfluenza viruses (PIV1-3) and human metapneumovirus (hMPV). Nasopharyngeal aspirates (NPAs) were collected from children with ALRI $\leq 5$ years of age. The sensitivity and specificity of mPCR was compared to virus isolation by centrifugation enhanced culture (CEC) followed by indirect immunofluorescence (IIF) (PreetiBharaj et al., 2009)

Smaller-scale influenza epidemics associated with antigenic drift contribute to mortality in the elderly and in those with pre-existing conditions such as chronic cardiopulmonary or renal disease, diabetes, immunosuppression or severe anaemia. The risk of Reye's syndrome is increased following influenza in children on long-term aspirin therapy. While the morbidity and excess mortality associated with influenza in temperate regions is well documented (Falsey et al., 2006), the more diffuse seasonality obscures the disease burden due to influenza in the tropics. Nevertheless, recent studies in Hong Kong and Singapore have revealed that influenza-associated mortality and morbidity in tropical settings is as significant as in temperate climates. Interestingly, influenza-associated mortality is not restricted solely to respiratory complications. A small proportion of cardiovascular mortality also appears to be triggered by influenza.

Avian influenza virus (H5N1) is currently endemic in poultry in a number of countries in Asia and Africa and has repeatedly been transmitted zoonotically to humans, often with fatal consequences (Peiris et al., 2007; AbdelGhafar et al., 2008).The associated disease was unusual in that previously healthy young adults and children are among those most severely ill. The disease presents as a rapidly progressive viral pneumonia with severe leucopenia and lymphopenia, progressing to Acute Respiratory Distress Syndrome and multi-organ dysfunction that fail to respond to standard antibiotic therapy for the pathogens causing community acquired pneumonia. Some patients also manifest a watery diarrhoea and moderate liver dysfunction. Most, though not all, patients have a history of recent exposure to sick poultry. Transmission remains zoonotic although occasional instances of limited and, so far, unsustained human-tohuman transmission following close family-type contact have been reported. Early diagnosis and treatment with oseltamivir is lifesaving. Irrespective of whether or not a putative pandemic threat becomes reality, it is clear that $\mathrm{H} 5 \mathrm{~N} 1$ viruses have already had a significant impact on the poultry industry, on human economic and social well-being and consequently on human health.

\section{HUMAN METAPNEUMOVIRUS}

This virus, which resembles Respiratory Syncytial 
Virus (RSV), was discovered in 2001 by van den Hoogen and colleagues in The Netherlands (Van Den Hoogen et al., 2003). It is now recognized to be a separate virus in its own right, although the disease it causes, its worldwide distribution and seasonality are similar to those of RSV (Fouchier et al., 2005; Banerjee et al., 2007). It may cause infections in the elderly as well as in babies under 1 year old and its discovery has accounted for some of the diseases in these age-groups for which no cause had been found hitherto. Retrospective serology, though, has shown that this is not a new pathogen, even for man, but has been around for a long time.

\section{PARAINFLUENZA VIRUS}

There are four serotypes of parainfluenza, type 1, 2, 3 and 4, with type 4 possessing two subtypes: 4 a and $4 \mathrm{~b}$. Types 1 and 2 typically cause croup, a high-pitched barking cough in children which is profoundly irritating. Type 3 can cause bronchiolitis or pneumonia and, less often, croup. In temperate countries, types 1 and 2 (together with RSV) are more prevalent in the winter months, whereas type 3 is unusual (among respiratory viruses) in occurring more often in spring and early summer. This dissociation between the peaks of activity of parainfluenza type 3 and RSV has also been observed in tropical regions (Jacob John et al., 1991).

\section{OTHER VIRUSES CAUSING DISEASE IN RESPIRATORY TRACT}

Human Boca viruses belong to the family Parvoviridae are associated with a proportion of lower respiratory tract disease and wheezing in children, especially those aged 6 months-2 years (Allander et al., 2007; Anderson, 2007).The viral DNA is also detectable in the serum but it is not clear whether this represents infectious virus. Enteroviruses have been known for many years as causes of a range of clinical manifestations. Their role in respiratory infections is now being increasingly investigated (Ruohola et al., 2006). Hantavirus pulmonary syndrome (HPS) is a rare but important cause of severe respiratory illness in the North and South American continents.

Their role in respiratory disease was first recognized in May 1993, when an outbreak of a severe and frequently fatal, respiratory disease occurred in the area in the USA where the four states Arizona, Colorado, New Mexico and Utah abut. The causative agent was found to be a hantavirus, later called SinNombre virus. The natural host was found to be the deer mouse, Peromyscusmaniculatus, the local population of which had recently increased rapidly, bringing them and their excreta more into contact with humans, and allowing the virus to cross the species gap. Related viruses causing a similar disease syndrome have since been isolated in North (e.g. New York, Bayou, Black Creek Canal viruses) and South (e.g. Andes virus) Americas, but with different species of natural rodent hosts. These viruses all belong to the same hanta virus genus as those causing haemorrhagic fever with renal syndrome (HFRS) in the Old World: Hantaan, Seoul and Puumala viruses. Both HFRS and HPS have a similar febrile prodrome with thrombocytopenia and leucocytosis. In HPS, the key differences are that the capillary leakage which follows is localized to the lungs and that, with Sin Nombrevirus, renal dysfunction is minimal. There was no evidence of human-to-human transmission in this outbreak, but there is evidence that some of the South American hantaviruses causing HPS may be transmitted between humans in a nosocomial setting.

\section{EPIDEMIOLOGY}

The aetiology and epidemiology of acute respiratory infections have been intensively studied in the temperate areas of the world. Information from tropical regions is more scanty, but what evidence there is suggests that the viruses responsible for respiratory disease in the tropics are no different from those found in temperate zones (Nokes et al., 2004; Jacob John et al., 1991; Bale et al., 1990). However, the severity of illness and its sequelae, as well as their seasonality, may be markedly different from those in the developing world (Shek et al., 2003). In temperate regions, respiratory infections have generally been shown to increase in the autumn and winter, although the exact mechanisms are still not fully understood. A similar periodicity is shown in tropical regions but this may be related to fluctuations in rainfall or humidity rather than temperature (Shek et al., 2003). In contrast to temperate regions, influenza in the tropics may occur in the summer months or all year round and RSV in subtropical Hong Kong is a summer disease.

The activities of influenza A and B remain impossible to predict and can fluctuate greatly from year to year. The appearance of a 'new' strain of either A or B can be associated with an epidemic the size of which is likely to be greater as the size of the antigenic change increases, although other, so far unidentified; virulence factors may be even more influential. With no shift changes in influenza B, major epidemics are less common. Even where high-quality, competent diagnostic services are available, not every clinical respiratory disease yields unequivocal evidence of infection by a virus or other microorganism. The proportion in which a positive diagnosis is made varies from a quarter to a half, depending on laboratory, area, population and time of year. The recent discoveries of a number of newly recognized respiratory viruses 
(e.g. human metapneumovirus, bocavirus, novel corona viruses and novel hantaviruses) has highlighted the fact that there are probably still more viruses to be uncovered. With the development and application of new methods to detect pathogens, novel respiratory viruses are likely to be increasingly recognized in the respiratory tract. It is essential, however, to differentiate asymptomatic viral carriage from infections of etiological significance, a task that requires careful epidemiological studies including the relevant controls (Jacob John et al., 1991).

\section{LABORATORY DIAGNOSIS}

There are three main reasons for providing a laboratory diagnosis of viral respiratory infections: for individual patient diagnosis to aid clinical management (specific therapy, stopping antibiotic therapy, infection control); to monitor routine virus activity in the community (epidemiology, e.g. vaccine strain selection for influenza); or for research investigations.

Rapid diagnosis of viral respiratory infections (i.e. in less than $3 \mathrm{~h}$ ) has been shown to reduce antibiotic use and to be cost effective (Woo et al., 1997). In addition, such confirmation of the cause is useful in hospital infection control (e.g. in cohorting similar cases) and occasionally, in deciding whether to use antiviral drugs in selected high-risk patients. The anti-neuraminidase drugs for treating influenza provide an additional incentive for making rapid diagnoses in, for example, an outbreak situation, although their cost may yet deter their widespread use.

It is self-evident that individual diagnosis must be quick if it is to influence clinical management. Diagnosis of respiratory infection is achievable within $2-3 \mathrm{~h}$ using techniques such as antigen detection (Gardner, 1980; Madeley, 1980; Arstila et al., 1988). However, these techniques are not universally available, even in hospitals in the developed world, mainly because they are labour and expertise-intensive. In the developing world this is compounded by a shortage of staff experienced in the use of such techniques, but these objections are surmountable. Enzyme immunoassays in 'kit format' which are relatively simple to perform are available for the diagnosis of influenza A and B and for Respiratory Syncytial Virus. They are, however, expensive. They have adequate positive and negative predictive value of infection during influenza epidemics but have poor predictive values during periods of low influenza activity (Grijalva et al., 2007). They also have poor sensitivity for the diagnosis of avian influenza (H5N1) disease in humans (Abdel-Ghafar AN et al., 2008).

The increasing need to provide diagnosis for avian influenza H5N1 which is best done by sensitive molecular (e.g. RT-PCR) methods (because other options are less sensitive and virus culture necessitates biosafety level 3 facilities) is increasing the need for establishing this technology in reference laboratories investigating respiratory infections, even in a developing country setting. This may in time permit more utilization of multiplex molecular tests for investigation of a wider range of respiratory pathogens (Multiplex PCR) (Freymuth et al., 2006). However, these methods remain resource and expertise intensive and need to be well controlled with regular quality control exercises. Microarray methods with the potential to detect a range of pathogens in a single test are in development on a research basis but it is unclear if they have adequate sensitivity for virus detection in clinical specimens (in contrast with virus isolates where high titres of nucleic acid are present). These methods however have potential for the detection of novel pathogens and also for greater recognition of co-infection by multiple pathogens.

\section{METHODS OF VIRAL DIAGNOSIS}

Laboratory diagnosis of respiratory virus infections depends on the demonstration of either virus or viral components in the patient at the acute stage of the illness, or subsequently an immune (serological) response to the virus.

\section{DEMONSTRATION OF VIRUS}

There are several approaches to this. They include demonstration of: (1) viral antigens by immunofluorescence (Gardner, 1980; Madeley., 1980) or enzyme immunoassays (Arstila et al., 1988) (2) viral infectivity by growth in cell culture; or (3) viral nucleic acid by various techniques. Virology specimens are perishable and must be delivered to the laboratory without delay. Methods of automated diagnosis depend on good-quality specimens being taken from the patient. It is easier to take a bad specimen than a good one, and close cooperation with the laboratory will help to raise the positivity rate.

\section{DEMONSTRATION OF IMMUNE RESPONSE}

This, at present, means demonstrating an antibody response in the serum to the stimulus provided by the virus. Seeking responses in cellular immunity or antibody in other body fluids remain research techniques only. For a valid diagnosis, a convalescent specimen of serum (taken after enough time for a response has elapsed) is needed but may be difficult to collect 2 weeks after the onset from patients who may by then be totally 
recovered and unwilling to oblige the investigator's interest. This is particularly true with children. Nevertheless, unless an antibody response can be demonstrated (seroconversion or a rising titre) some uncertainty over the validity of the result will remain. The alternative is to demonstrate an IgM-class response but this suffers from the twin disadvantages that such tests are not available for all viruses and the sample (to be reliably positive) may have to be taken after the acute illness is over, with the problem(s) already mentioned.

Serology remains the routine choice for some respiratory agents which, although not viral in nature, are traditionally diagnosed by virus laboratories. These include: psittacosis, Q fever and Mycoplasmapneumoniaeinfection. All cause an illness with an insidious onset and are difficult and/or dangerous to isolate. Since all, therefore, are susceptible to antibiotics, a diagnosis is important and can be life-saving.

\section{TREATMENT}

Amantadine and its alternative rimantidine, were options for the prevention (in outbreaks within closed communities of high risk individuals) and less convincingly for the treatment of influenza A (Hayden et al., 2006). However, since 2003, increasing resistance of both H3N2 and H1N1 subtypes of influenza A virus has now led to its withdrawal as an option for the treatment of seasonal influenza. Neuraminidase inhibitors such as zanamivir and oseltamivir, which inhibit the viral enzyme neuraminidase from both influenza $\mathrm{A}$ and $\mathrm{B}$ (concerned with release of the virus from infected cells) are effective for the treatment and prophylaxis of influenza but have to be given within $48 \mathrm{~h}$ of onset for apparent clinical benefit. They are expensive and are best used on those most at risk of serious illness- those at the extremes of life. They are also effective against other influenza A virus subtypes including avian influenza A H5N1. While either zanamivir (given by inhaler) or oseltamivir (given orally) can be used for prophylaxis of $\mathrm{H} 5 \mathrm{~N} 1$ influenza disease, given the potential for dissemination of this virus beyond the respiratory tract, the systemically active oseltamivir is the preferred option for treatment.

However, experience with human cases of H5N1 avian influenza has shown that resistance develops rapidly and may be a major problem in widespread prophylactic or therapeutic use. These drugs are not active on other respiratory viruses, even those with viral neuraminidases. Generally, the management of viral respiratory infections is essentially symptomatic. Antibiotics are not routinely indicated for viral respiratory infections unless secondary bacterial super infection occurs. The 'atypical' bacte- rial infections mentioned above $(\mathrm{Q}$ fever, mycoplasmosis and chlamydiosis) are amenable to antibiotic therapy. (T) ribavirin given as an aerosol inhalation is claimed to reduce the severity of RSV infection in infants, but this remains controversial. It is a very expensive drug, but may be life-saving in those with congenital heart and/or lung damage for whom RSV infection may be the final insult which pushes them into heart or lung failure. (T) ribavirin may have some effect in influenza but the evidence is minimal. It has also been used in hantavirus pulmonary syndrome but, again, the evidence of efficacy is minimal. Aciclovir (given i.v.) is effective in the treatment of varicella or herpes simplex infections of the respiratory tract in the immuno compromised patient. It should also be used in an immunocompetent patient (usually an adult) with varicella pneumonia. Gancyclovir and foscarnet are useful in cytomegalovirus infection in the immunosuppressed.

\section{DIAGNOSIS}

In the last decade, Several viruses are newly discovered including human metapneumovirus (hMPV), coronaviruses NL63 (HcoV-NL63) and HKU1 (HcoV-HKU1), Human Bocavirus (HBoV), new enterovirus (HEV), parechovirus (HpeV) and rhinovirus (HRV) strains, polyomaviruses WU (WUPyV) and KI (KIPyV) and the pandemic H1N1v influenza A virus.(Maurizia Debiaggi et al., 2012; SuatBicer et al., 2013)

A RT-PCR assay was used for detection of Human Viruses. 143 respiratory specimens from 126 patients was analyzed through RT-PCR. Results are compared with other methods RT-PCR proved to be positive for 17 of 143 specimens. It shows that RT-PCR provides high sensitivity than other diagnostic method (Lisa Liolios et al., 2001)

Diagnosis of respiratory syncytial virus (RSV) during acute infection in adults is difficult because of the poor sensitivity of viral culture and antigen detection. A recently developed single-tube nested reverse transcription-PCR (RT-PCR) was compared to viral culture and serology by enzyme immunoassay for the diagnosis of RSV in adults with respiratory illness. Nasal swab samples were collected during respiratory illnesses from five groups of subjects: healthy young adults, healthy elderly adults, adults with chronic heart and lung disease, nursing home residents, and adults admitted to the hospital during the winter with cardiopulmonary illnesses. Of 1,112 samples for which all three tests were available, 117 were positive by at least one method and 995 were negative by all methods. (Ann Falsey et al., 2002; Mahony et al., 2007)

Laboratory diagnosis of human respiratory syncytial 
virus (hRSV) infections has traditionally been performed by virus isolation in cell culture and the direct fluorescent-antibody assay (DFA). Reverse transcriptase PCR (RT-PCR) is now recognized as a sensitive and specific alternative for detection of hRSV in respiratory samples. Using the Light Cycler instrument, a rapid RT-PCR assay for the detection of hRSV (the LC-RT-PCR) with a pair of hybridization probes that target the hRSV L gene was developed. The sensitivity of LC-RT-PCR was 50 PFU/ $\mathrm{ml}$; and this, together with its high specificity and rapid turnaround time, makes the LC-RT-PCR suitable for the detection of hRSV in clinical specimens (David Whiley et al., 2002)

A nucleic acid-based assay that is rapid, sensitive, and specific can be used for the simultaneous detection of five common human respiratory pathogens, including influenza virus A, influenza virus B, parainfluenza virus types 1 and 3, respiratory syncytial virus (RSV), and adenovirus groups B, C, and E. Typically, diagnosis on an unextracted clinical sample can be provided in less than $3 \mathrm{~h}$, including sample collection, preparation, processing as well as data analysis. Such a multiplexed panel would enable rapid broad-spectrum pathogen testing on nasal swabs and therefore allow implementation of infection control measures and the timely administration of antiviral therapies (Sonia Le'tant et al., 2007).

Respiratory samples from hospitalized patients with respiratory symptoms were tested by real-time PCR, viral culture and direct immunofluorescence for respiratory syncytial virus, influenza virus, parainfluenza viruses, and adenoviruses. Compared with conventional diagnostic tests, real-time PCR increased the diagnostic yields for these viruses from $24 \%$ to $43 \%$ and from $3.5 \%$ to $36 \%$ for children and adults, respectively (Alma van de Pol et al., 2007). Immune functions were evaluated in vitro for PBMC isolated from healthy donors and cultured with the antiviral agents, 3'-azido-3'-deoxythymidine (AZT), ribavirin, ganciclovir, 2'3'-dideoxyinosine (ddI), or acyclovir (Berman, et al., 1991).

Influenza virus and respiratory syncytial virus (RSV) are the predominant etiological agents during seasonal epidemics and thus rapid and sensitive molecular tests for screening for such agents and timely identification of epidemics are required. The study compares real-time quantitative PCR (qPCR) with conventional RT-PCR for parallel identification of influenza A virus (IAV) or influenza B virus (IBV) and RSV (Anurodh Agrawal et al., 2009). Hospital and community-based studies of ALRI have found that measles accounts for $6-21 \%$ of the morbidity and $8-93 \%$ of the mortality due to ALRI ( Markowitz LE et al.,1991).

A multiplex reverse transcription (RT)-PCR method that has been developed is capable of detecting and sub typing influenza A (H1N1 and H3N2) and B viruses as well as respiratory syncytial virus (RSV) types A and B in respiratory clinical samples taken as part of a national community-based surveillance program of influenza-like illness in England and Wales. The detection of each different pathogen depended on distinguishing five amplification products of different sizes on agarose gels following RT-PCR with multiple primer sets (Stockton et al., 1998).

Clinical presentations for viral respiratory tract infections are often nonspecific and a rapid, high throughput laboratory technique that can detect a panel of common viral pathogens is clinically desirable. Two multiplex reverse transcription-PCR (RT-PCR) products coupled with microarray-based systems for simultaneous detection of common respiratory tract viral pathogens were detected. The NGEN respiratory virus analyte-specific assay (Nanogen, San Diego, CA) detects influenza A virus (Flu-A) and Flu-B, parainfluenza virus 1 (PIV-1), PIV-2, and PIV-3, and respiratory syncytial virus (RSV), while the ResPlex II assay (Genaco Biomedical Products, Inc., Huntsville, AL) detected Flu-A, Flu-B, PIV-1, PIV-2, PIV-3, PIV-4, RSV, human metapneumovirus (hMPV), rhinoviruses (RhVs), enteroviruses (EnVs) and severe acute respiratory syndrome (SARS), coronavirus (CoV). The two systems processed 80 (NGEN) and 96 (ResPlex II) tests per run, with a hands-on time of approximately $60 \mathrm{~min}$ and test turnaround times of $6 \mathrm{~h}$ (ResPlex II) and 9 h (NGEN). Multiple-panel testing detected an additional unsuspected 9 (3.4\%) PIV-1 and 10 (3.7\%) PIV-3 infections. Both the NGEN and ResPlex II assays provided userfriendly and high-throughput tools for simultaneous detection and identification of a panel of common respiratory viral pathogens in a single test format. The multiplex approach enhanced diagnosis through detection of respiratory viral etiological agents in cases in which the presence of the agent was not suspected and a test was not ordered by the clinicians (Haijing Li et al., 2007).

Evaluation of the Prodesse ProFlu-1 real-time reverse transcription-PCR multiplex assay with the Smart-Cycler instrument for the detection of human respiratory syncytial virus (RSV) and influenza A and B viruses in comparison to conventional cell culture and antigen immunoassays with the BD Directigen A_B and Binax was done. Ninety-two percent of the 361 specimens tested were nasopharyngeal aspirates obtained from individual patients, of which 119 were positive for RSV and 59 were positive for influenza virus. The median age of the patients whose specimens were positive for RSV and influenza virus were 6.3 months and 42.4 years, respectively (Liao et al., 2009).

The etiology of lower respiratory tract infections 
in young children admitted to the pediatric intensive care unit (PICU) is often difficult to establish. However, most infections are believed to be caused by respiratory viruses. A diagnostic study was performed to compare conventional viral tests with the recently developed real-time PCR technique. Real-time PCR for respiratory viruses was found to be a sensitive and reliable method in PICU patients with lower respiratory tract infection, increasing the diagnostic yield two fold compared to conventional methods (Alma van de Pol et al., 2006).

The multiplex PCR experiment is to amplify multiple regions of a DNA sequence at the same time by using different primer pairs. Designing feasible primer pairs for multiplex PCR is a tedious task since there are too many constraints to be satisfied. A new method for multiplex PCR primer design strategy using genetic algorithm was proposed. The proposed algorithm was able to find a set of suitable primer pairs more efficient and uses a MAP model to speed up the examination of the specificity constraint that is important for gene family sequences. The dry-dock experiment shows that the proposed algorithm finds several sets of primer pairs of gene family sequences for multiplex PCR that not only obey the design properties, but also have specificity (Hong-Long Liang et al., 2005).

Human adenovirus (HAdV) is an important agent causing respiratory tract infection in children. Information on the epidemiological and clinical features of HAdV is limited in children with acute respiratory tract infections (ARTIs) in China, especially those of a novel genotype, Ad55. HAdV is an important viral agent in children with ARTIs in Lanzhou City, China. Multiple HAdV serotypes co-circulated with Ad3, which was predominant in the 3-year study. The novel AdV55 genotype was found in one case. No fixed seasonal rhythm was identified (Yu Jin et al., 2013).

Severe lower respiratory infections (LRIs) and atopic sensitization have been identified as independent risk factors for asthma. The study suggests that viral infections interact with atopy in infancy to promote later asthma. Notably the occurrence of both of these events during this narrow developmental window was associated with maximal risk for subsequent asthma, which suggests a contribution from both classes of inflammatory insults to disease pathogenesis. Protection of "highrisk" children against the effects of severe respiratory infections during infancy may represent an effective strategy for primary asthma prevention. The potential benefits of these strategies merit more careful evaluation in this age group (Merci Kusel et al., 2007).

This study was performed to evaluate the associations of newly recognized viruses, namely, human metapneu- movirus (hMPV), human corona virus (HCoV)-NL63 and human bocavirus $(\mathrm{HBoV})$ with lower respiratory tract infections (LRTIs) in previously healthy children (EunHwa Choi et al., 2006).

Highly pathogenic avian influenza virus of the subtype H5N1 may cause infection of the lower respiratory tract (LRT) and severe pneumonia in humans. However, the cell types in the LRT to which the virus attaches are unknown for both humans and experimental animals. Although attachment is not the only factor required for virus replication, this information is important both to better understand the pathogenesis of H5N1 influenza and to assess the suitability of animal models. Therefore, a comparison was made between the pattern of H5N1 virus attachment to the LRT of humans and four animal species (Debby van Riel et al., 2006).

A novel corona virus $(\mathrm{SCOV})$ is the etiological agent of severe acute respiratory syndrome (SARS). SCoV-like viruses were isolated from Himalayan palm civets found in a live-animal market in Guangdong, China. Evidence of virus infection was also detected in other animals (including a raccoon dog, Nyctereutesprocyonoides) and in humans working at the same market. All the animal isolates retain a 29-nucleotide sequence that is not found in most human isolates. The detection of SCoV-like viruses in small, live wild mammals in a retail market indicates a route of interspecies transmission, although the natural reservoir is not known (Guan et al., 2003).

Influenza and respiratory syncytial virus (RSV) cause substantial morbidity and mortality. Statistical methods used to estimate deaths in the United States attributable to influenza have not accounted for RSV circulation. Influenza deaths have increased substantially in the last 2 decades, in part because of aging of the population, underscoring the need for better prevention measures, including more effective vaccines and vaccination programs for elderly persons (William Thompson et al., 2003).

In 2009, officials at the Centers for Disease Control and Prevention (CDC) confirmed two cases of swine influenza in children living in neighboring counties in California. A perspective from systems biology is taken to review the series of evolutionary and epidemiologic events, starting in 1918, that led to the emergence of the current swine-origin influenza A (H1N1) strain (S-OIV), which is widely known as swine flu (Shanta Zimmer et al., 2009).

In Hong Kong, the reason for SARS was found to be mainly contact in households and healthcare settings, putting children at particular risk. Most data so far, however, have been for adults. Prospective follow up of the first ten children with SARS were managed during the early phase of the epidemic in Hong Kong. All the children had been in close contact with infected adults. 
Persistent fever, cough, progressive radiographic changes of chest and lymphopenia were noted in all patients. The children were treated with high-dose ribavirin, oral prednisolone, or intravenous methylprednisolone, with no short-term adverse effects. Four teenagers required oxygen therapy and two needed assisted ventilation. None of the younger children required oxygen supplementation. Compared with adults and teenagers, SARS seems to have a less aggressive clinical course in younger children (Hon et al., 2003).

In 1997, H5N1 influenza A virus was transmitted from birds to humans in Hong Kong, killing 6 of the 18 people infected. When mice were infected with the human isolates, two virulence groups became apparent. Using reverse genetics, it showed that a mutation at position 627 in the PB2 protein influenced the outcome of infection in mice. Moreover, high cleavability of the hemagglutinin glycoprotein was an essential requirement for lethal infection (Masato Hatta et al., 2001).

\section{CONCLUSION}

The detection rate of co-infections was similar to previously reported multiplex PCR studies. It was observed that higher percentage of children with mixed infections had severe and very severe ALRI as compared to ALRI. Previous studies have shown that co-infection with different respiratory viruses might lead to a more severe disease or multiple viruses have been detected from patients with severe disease as described LRI caused by RSV was more common in younger children as reported earlier. RSV and HmPV were associated with bronchiolitis. PIVs and Influenza viruses were associated with pneumonia similar to previous findings. However, the number of all the viral detections except RSV was too few to comment on the association of the virus with bronchiolitis or pneumonia.

\section{REFERENCES}

1. Allander T, Jartti T, Gupta S (2007). Human bocavirus and cute wheezing in children. Clin Infect Dis. 44:904-910.

2. Alma C van de Pol, Tom FW Wolfs, Nicolaas JG Jansen, Anton M van Loon, John WA Rossen, (2006). Diagnostic value of real-time polymerase chain reaction to detect viruses in young children admitted to the pediatric intensive care unit with lower respiratory tract infection. BioMed Central Ltd.

3. Anderson LJ (2007). Human bocavirus: a new viral pathogen. Clin Infect Dis. 44: 911-912.

4. Ann R. Falsey, Maria A. Formica, and Edward E. Walsh (2002). Diagnosis of Respiratory Syncytial Virus Infection: Comparison of Reverse Transcription-PCR to Viral Culture and Serology in Adults with Respiratory Illness. J. ClinMicrobiol. 40:817-820.

5. Anurodh S Agrawal, MehuliSarkar, SekharChakrabarti, K Rajendran, HarpreetKaur, Akhilesh C Mishra, Mrinal
K Chatterjee, Trailokya N Naik, Mandeep S Chadha and MamtaChawla-Sarkar (2009). Comparative evaluation of real-time PCR and conventional RT-PCR during a 2 year surveillance for influenza and respiratory syncytial virus among children with acute respiratory infections in Kolkata, India. Journal of Medical Microbiology. 58:1616-1622.

6. Darwyn Kobasa, Steven M Jones, Kyoko Shinya, John C Kash, John Copps, Hideki Ebihara, Yasuko Hatta, Jin Hyun Kim, Peter Halfmann, Masato Hatta, Friederike Feldmann, Judie B Alimonti, Lisa Fernando, Yan Li1, Michael G. Katze, Heinz Feldmann\& Yoshihiro Kawaoka (2007). Aberrant innate immune response in lethal infection of macaques with the 1918 influenza virus. Nature. 445:78-90.

7. David M Whiley, Melanie W Syrmis, Ian M Mackay and Theo P Sloots (2002). Detection of Human Respiratory Syncytial Virus in Respiratory Samples by Light Cycler Reverse Transcriptase PCR. J. Clin. Microbiol. 44:18-23.

8. Debby van Riel, Vincent J Munster, Emmie de Wit, Guus F Rimmelzwaan, Ron AM Fouchier, Ab DME Osterhaus, ThijsKuiken (2006). H5N1 Virus Attachment to Lower Respiratory Tract. Science. 312:89-96.

9. EunHwa Choi, Hoan Jong Lee, Sun Jung Kim, ByungWookEun, Nam Hee Kim, Jin A Lee, Jun Ho Lee, Eun Kyung Song, So Hee Kim Ji Yong Park, and JiYeon Sung (2006). The Association of Newly Identified Respiratory Viruses with Lower Respiratory Tract Infections in Korean Children, 2000-2005. New Respiratory Viruses in Children. 43:34-45.

10. Feng-Mao Lin, Hsien-Da Huang, His-Yuan Huang,JorngTzongHorng (2005). Primer Design for Multiplex PCR Using a Genetic Algorithm. Gecco'05. 45:78-89.

11. George Quellhorst and Sam Rulli (2000). A Systematic Guideline for Developing the Best Real-Time PCR Primers RT2 Profiler PCR Arrays.

12. Haijing Li, Melinda A. McCormac, R. Wray Estes, Susan E. Sefers, Ryan K. Dare, James D. Chappell, Dean D. Erdman, Peter F. Wright, and Yi-Wei Tang (2007). Simultaneous Detection and High-Throughput Identification of a Panel of RNA Viruses Causing Respiratory Tract Infections. J. ClinMicrobiol. 45:2105-2109.

13. Hong-Long Liang, Chungnan Lee, Jain-Shing Wu (2009). Multiplex PCR Primer Design for Gene Family Using Genetic Algorithm. Gecco'05. 45:67-78.

14. Mahony, S Chong, F Merante, S Yaghoubian, T Sinha, C Lisle and R Janeczko (2007). Development of a Respiratory Virus Panel Test for Detection of Twenty Human Respiratory Viruses by Use of Multiplex PCR and a Fluid Microbead-Based Assay. J. ClinMicrobiol. 45:29-35.

15. Stockton, JS Ellis, M Saville, JP Clewley and MC Zambon (1998). Multiplex PCR for Typing and Subtyping Influenza and Respiratory Syncytial Viruses. J. ClinMicrobiol. 36:2990-2995.

16. Hayden FG, B Winther, GR Donowitz, SE Mills and DJ Innes (1987). Human nasal mucosal responses to topically applied recombinant leukocyte A interferon. J. Infect. Dis. 156:64-72.

17. Hayden FG, DL Kaiser and JK Albrecht (1988). Intranasal recombinant alfa- $2 \mathrm{~b}$ interferon treatment of naturally occurring common colds. Antimicrob. Agents Chemother. 32:224-230.

18. Hayden FG, RB Turner, JM Gwaltney, K Chi-Burris, M Gersten, P Hsyu, AK Patick, GJ Smith, 3rd, and LS Zalman (2003). Phase II, randomized, double-blind, placebocontrolled studies of ruprintrivir nasal spray 2-percent suspension for prevention and treatment of experimen- 
tally induced rhinovirus colds in healthy volunteers. $A n$ timicrob. Agents Chemother. 47:3907-3916.

19. Heagy W, C Crumpacker, PA Lopez and RW Finberg (1991). Inhibition of immune functions by antiviral drugs. J. Clin. Investig. 87:1916-1924.

20. Hon, CW Leung, WTF Cheng, PKS Chan, WCW Chu, YW Kwan, AM Li, NC Fong, PC Ng, MC Chiu, CK Li, JS Tam, TF Fok (2003). Clinical presentations and outcome of severe acute respiratory syndrome in children. The Lancet. 36:1702.

21. Liao, LL Tomalty, A Majury and DE Zoutman (2009). Comparison of Viral Isolation and Multiplex Real-Time Reverse Transcription-PCR for Confirmation of Respiratory Syncytial Virus and Influenza Virus Detection by Antigen Immunoassays. J. ClinMicrobiol. 47:527-532.

22. Lisa Liolios, Adam Jenney, Denis Spelman, Tom Kotsimbos, Michael Catton and Steve Wesselingh (2001). Comparison of a Multiplex Reverse Transcription-PCREnzyme Hybridization Assay with Conventional Viral Culture and Detection of Seven Viral RespiratoryPathogens. J. ClinMicrobiol. 39:2779.

23. Masato Hatta, PengGao, Peter Halfmann, Yoshihiro Kawaoka (2001). Molecular Basis for High Virulence of Hong Kong H5N1 Inßuenza A Viruses. Science. 293: 1840.

24. Maurizia Debiaggi, Filippo Canducci, Elisa Rita Ceresola and Massimo Clementi (2012). The role of infections and co-infections with newly identified and emerging respiratory viruses in children. Virology Journal. 9:247.

25. Merci MH Kusel, Nicholas H de Klerk, Tatiana Kebadze, VaikeVohma, Patrick G. Holt, Sebastian L. Johnston and Peter D (2007). Early-life respiratory viral infections, atopic sensitization, and risk of subsequent development of persistent asthma. J Allergy Clin Immunol. 331:1106.

26. Markowitz LE, Nieburg P (1991). The burden of acute respiratory infection due to measles in developing countries and the potential impact of measles vaccine. Rev Infect Dis. 13:S555-S561.

27. Nokes DJ, Okiro EA, Ngama M (2004). Respiratory syncytial virus epidemiology in a birth cohort from Kilifi district, Kenya. J Infect Dis. 190:1828-1832.

28. Preeti Bharaj, Wayne M Sullender, Sushil K Kabra, KalaivaniMani, JohnCherian, VikasTyagi, Harendra S Chahar1, Samander Kaushik, Lalit Dar and Shobha Broor (2009). Respiratory viral infections detected by multiplex
PCR amongpediatric patients with lower respiratory tract infections seen at anurban hospital in Delhi from 2005 to 2007. Virology Journal. 6:89.

29. Ruslan Kalender, David Lee, Alan H Schulman (2009). Fast PCR Software for PCR Primer and Probe Design and Repeat Search, Genes, Genomes and Genomics. Global Science Books. 3:114.

30. Shanta M Zimmer and Donald S Burke (2009). Historical Perspective Emergence of Influenza A (H1N1). Viruses. 3:361.

31. Sonia E Le'tant, Josue I Ortiz, Lance F Bentley Tammero, James M Birch, Robert W Derlet, Stuart Cohen, Dannelle Manning and Mary T McBride (2007). Multiplexed Reverse Transcriptase PCR Assay for Identification of Viral Respiratory Pathogens at the Point of Care. J. ClinMicrobiol. 45:3498-3505.

32. SuatBicer, Tuba Giray, DefneÇöl, GülayÇilerErdağ, AyçaVitrinel, YesimGürol, GüldenÇelik, ÇigdemKaspar and ÖznurKüçük (2013). Virological and clinical characterizations of respiratory infections in hospitalized children. Italian Journal of Pediatrics. 39:22.

33. William W Thompson, David K Shay, Eric Weintraub, Lynnette Brammer, Nancy Cox, Larry J. Anderson, Keiji Fukuda (2003). Mortality Associated With Influenza and Respiratory Syncytial Virus in the United States. American Medical Association. JAMA 289:2-179.

34. Y Guan, BJ Zheng, YQ He, XL Liu, ZX Zhuang, CL Cheung, SW Luo, PH Li, LJ Zhang, YJ Guan, KM Butt, KL Wong, KW Chan, W Lim, KF Shortridge, KY Yuen, JSM Peiris, LLM Poon (2003). Isolation and Characterization of Viruses Related to the SARS Coronavirus from Animals in Southern China. Science. 302:276.

35. Yu Jin, Rong-fang Zhang, Zhi-ping Xie, Kun-long Yan, Han-chunGao, Jing-rong Song, Xin-hui Yuan, Yun-de Hou and Zhao-junDuan (2013). Prevalence of adenovirus in children with acute respiratory tract infection in Lanzhou, China. Virology Journal. 10:271.

How to cite article: P.Sagadeven, R.Ramya, S.Rathishkumar, S.Vijaykumar, L.Surendran, P.Janarthanan and C.Sajith (2018). Overview on common respiratory virus. Int J App Pharm Sci Res. 3(3):24-32. doi: https://doi.org/10.21477/ ijapsr.3.3.1

Source of Support; Nil Conflict of Interest: None declared 